\title{
Coupling hydrothermal treatment with stripping technology for fast ammonia release and effective nitrogen recovery from chicken manure
}

Weiwei Huang, ${ }^{\dagger, \star}$ Tian Yuan, ${ }^{\dagger, *}$ Ziwen Zhao, ${ }^{\dagger}$ Xi Yang, ${ }^{\dagger}$ Wenli Huang, ${ }^{\S}$ Zhenya Zhang, ${ }^{, * \dagger}$ and Zhongfang Lei ${ }^{*, \dagger}$

${ }^{\dagger}$ Graduate School of Life and Environmental Sciences, University of Tsukuba, 1-1-1 Tennodai, Tsukuba, Ibaraki 305-8572, Japan

${ }^{\S}$ MOE Key Laboratory of Pollution Process and Environmental Criteria, College of Environmental Science and Engineering, Nankai University, Tianjin 300071, China

\section{Corresponding Authors}

*Z. Zhang. Tel./fax: +81 298534712. Email: zhang.zhenya.fu@u.tsukuba.ac.jp.

*Z. Lei. Tel./fax: +81 298536703. Email: lei.zhongfang.gu@u.tsukuba.ac.jp.

${ }^{\ddagger}$ These authors contributed equally to this paper. 


\section{Tables}

Table S1. Calculation of dry matter loss and nitrogen recovery efficiency through HTT process.

\begin{tabular}{ccccccccc}
\hline \multirow{2}{*}{ Sample } & \multicolumn{2}{c}{ HTT conditions } & & & & & & Measured \\
\cline { 2 - 6 } & $\begin{array}{c}\text { Temperature } \\
\left({ }^{\circ} \mathrm{C}\right)\end{array}$ & $\begin{array}{c}\text { Holding } \\
\text { time } \\
(\mathrm{min})\end{array}$ & $\begin{array}{c}\text { DM in } \\
\mathrm{CM}(\mathrm{mg})\end{array}$ & $\begin{array}{c}\text { Organic-N } \\
\text { in } \mathrm{CM} \\
(\mathrm{mg})\end{array}$ & $\begin{array}{c}\text { Loss of } \\
\mathrm{DM} \\
(\mathrm{mg})\end{array}$ & $\begin{array}{c}\text { Loss of } \\
\text { organic-N } \\
(\mathrm{mg})\end{array}$ & $\begin{array}{c}\text { TAN } \\
\text { release } \\
(\mathrm{mg})\end{array}$ & $\begin{array}{c}\text { recovery } \\
\text { efficiency } \\
(\%)\end{array}$ \\
\hline $\mathrm{RCM}$ & - & - & 32500.00 & 444.28 & - & - & - & - \\
$180-15^{*}$ & 180 & 15 & 29372.00 & 233.11 & 3128.00 & 211.17 & 187.99 & 89.02 \\
$180-30$ & 180 & 30 & 28566.06 & 198.43 & 3933.94 & 245.85 & 206.72 & 84.08 \\
$180-60$ & 180 & 60 & 27624.96 & 156.91 & 4875.04 & 287.37 & 241.95 & 84.19 \\
$220-0$ & 220 & 0 & 24089.11 & 128.99 & 8410.89 & 315.29 & 259.80 & 82.40 \\
\hline
\end{tabular}

DM-dry matter, TAN-total ammonia nitrogen, RCM-raw chicken manure. *a-b denotes that the HTT process was performed at peak temperature of $a$ for $b$ min. 
Table S2. Correlation coefficient matrix between two tested parameters in the processed CM under different HTT conditions $(\mathrm{n}=12)$.

\begin{tabular}{|c|c|c|c|c|c|c|c|c|c|c|}
\hline Parameters & $\begin{array}{l}\text { TAN } \\
(\mathrm{mg} / \mathrm{g}-\mathrm{VS})\end{array}$ & $\begin{array}{l}\text { TS reduction } \\
(\%)\end{array}$ & $\begin{array}{l}\text { VS reduction } \\
(\%)\end{array}$ & $\begin{array}{l}\text { SOC } \\
(\mathrm{mg} / \mathrm{g}-\mathrm{VS})\end{array}$ & $\begin{array}{l}\text { Proteins-C } \\
(\mathrm{mg} / \mathrm{g}-\mathrm{VS})\end{array}$ & $\begin{array}{l}\text { Carbohydrates-C } \\
(\mathrm{mg} / \mathrm{g}-\mathrm{VS})\end{array}$ & $\begin{array}{l}\text { TVFAs-C } \\
(\mathrm{mg} / \mathrm{g}-\mathrm{VS})\end{array}$ & $\begin{array}{l}\mathrm{CO}_{2} \text { yield } \\
(\mathrm{ml} / \mathrm{g}-\mathrm{VS})\end{array}$ & $\mathrm{C} / \mathrm{N}$ & $\mathrm{H} / \mathrm{N}$ \\
\hline TAN (mg/g-VS) & 1 & $0.885^{* *}$ & $0.916^{* *}$ & NS & NS & NS & $0.932^{* *}$ & $0.888^{* *}$ & $-0.877^{* *}$ & $-0.970^{* *}$ \\
\hline TS reduction $(\%)$ & & 1 & $0.980^{* *}$ & NS & NS & NS & $0.921^{* *}$ & $0.976^{* *}$ & $-0.707^{*}$ & $-0.888^{* *}$ \\
\hline VS reduction $(\%)$ & & & 1 & NS & NS & NS & $0.945^{* *}$ & $0.971^{* *}$ & $-0.701^{*}$ & $-0.894^{* *}$ \\
\hline $\mathrm{SOC}(\mathrm{mg} / \mathrm{g}-\mathrm{VS})$ & & & & 1 & $0.850^{* *}$ & $0.986^{* *}$ & NS & NS & NS & NS \\
\hline Proteins-C (mg/g-VS) & & & & & 1 & $0.890^{* *}$ & NS & $-0.636^{*}$ & NS & NS \\
\hline Carbohydrates-C (mg/g-VS) & & & & & & 1 & NS & NS & NS & NS \\
\hline TVFAs-C (mg/g-VS) & & & & & & & 1 & $0.929^{* *}$ & $-0.721^{* *}$ & $-0.875^{* *}$ \\
\hline $\mathrm{CO}_{2}$ yield $(\mathrm{ml} / \mathrm{g}-\mathrm{VS})$ & & & & & & & & 1 & $-0.696^{*}$ & $-0.868^{* *}$ \\
\hline $\mathrm{C} / \mathrm{N}$ & & & & & & & & & 1 & $0.937^{* *}$ \\
\hline $\mathrm{H} / \mathrm{N}$ & & & & & & & & & & 1 \\
\hline
\end{tabular}

NS-No significance. ${ }^{*} \mathrm{p}<0.05$, and ${ }^{*} \mathrm{p}<0.01$. TAN-total ammonia nitrogen, TS-total solids, VS-volatile solids, SOC-soluble organic carbon, TVFAs-total volatile fatty acids. 
Table S3. Initial $\mathrm{CH}_{4}$ fermentation conditions.

\begin{tabular}{|c|c|c|c|c|c|}
\hline \multirow{2}{*}{ Reactor } & \multicolumn{2}{|c|}{ HTT conditions } & \multicolumn{3}{|c|}{ Initial fermentation conditions } \\
\hline & $\begin{array}{c}\text { Temperature } \\
\left({ }^{\circ} \mathrm{C}\right)\end{array}$ & $\begin{array}{l}\text { Holding time } \\
\text { (min) }\end{array}$ & TS $(\%)$ & VS $(\%)$ & TAN (mg/L) \\
\hline $\mathrm{RCM}$ & - & - & $6.99 \pm 0.32$ & $4.44 \pm 0.15$ & $402.81 \pm 4.64$ \\
\hline HTT180-15 & 180 & 15 & $6.68 \pm 0.24$ & $3.97 \pm 0.17$ & $130.32 \pm 2.09$ \\
\hline HTT180-30 & 180 & 30 & $6.58 \pm 0.19$ & $3.89 \pm 0.20$ & $131.84 \pm 5.02$ \\
\hline HTT180-60 & 180 & 60 & $6.57 \pm 0.21$ & $3.89 \pm 0.18$ & $119.05 \pm 5.11$ \\
\hline HTT220-0 & 220 & 0 & $6.11 \pm 0.21$ & $3.32 \pm 0.15$ & $108.89 \pm 4.17$ \\
\hline
\end{tabular}

Initial manure $\mathrm{pH}$ was adjusted to around 7.0. RCM-raw chicken manure without hydrothermal treatment and ammonia stripping, HTT-hydrothermal treatment, TS-total solids, VS-volatile solids, TAN-total ammonia nitrogen. 


\section{Scheme}

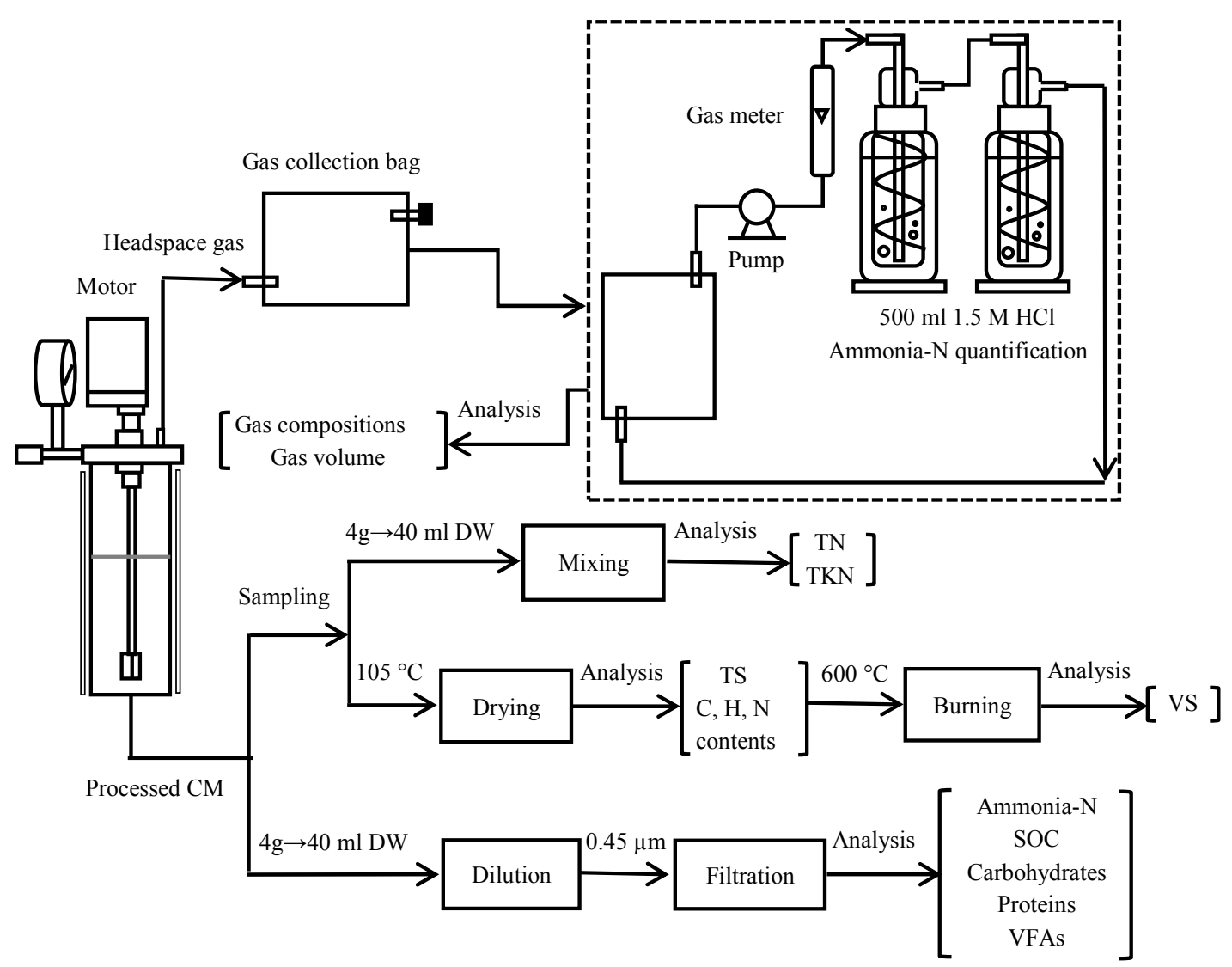

CM-chicken manure, TS-total solids, VS-volatile solids, DW-deionized water, TKN-total Kjeldahl nitrogen, TN-total nitrogen, SOC-soluble organic carbon, VFAs-volatile fatty acids.

Scheme S1. Flowchart of analytical procedures during HTT experiments. 


\section{Figures}

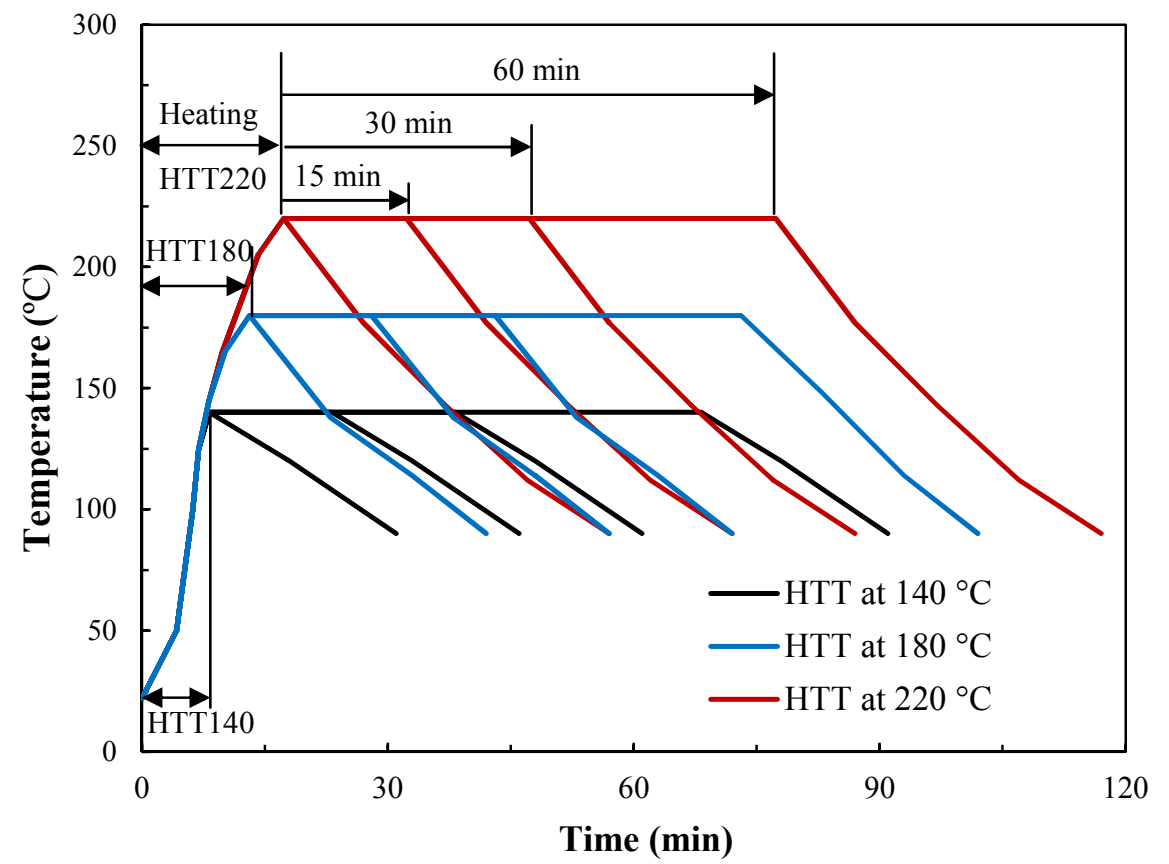

Fig. S1. Variations of temperature in the HTT reactor under different operational conditions. 


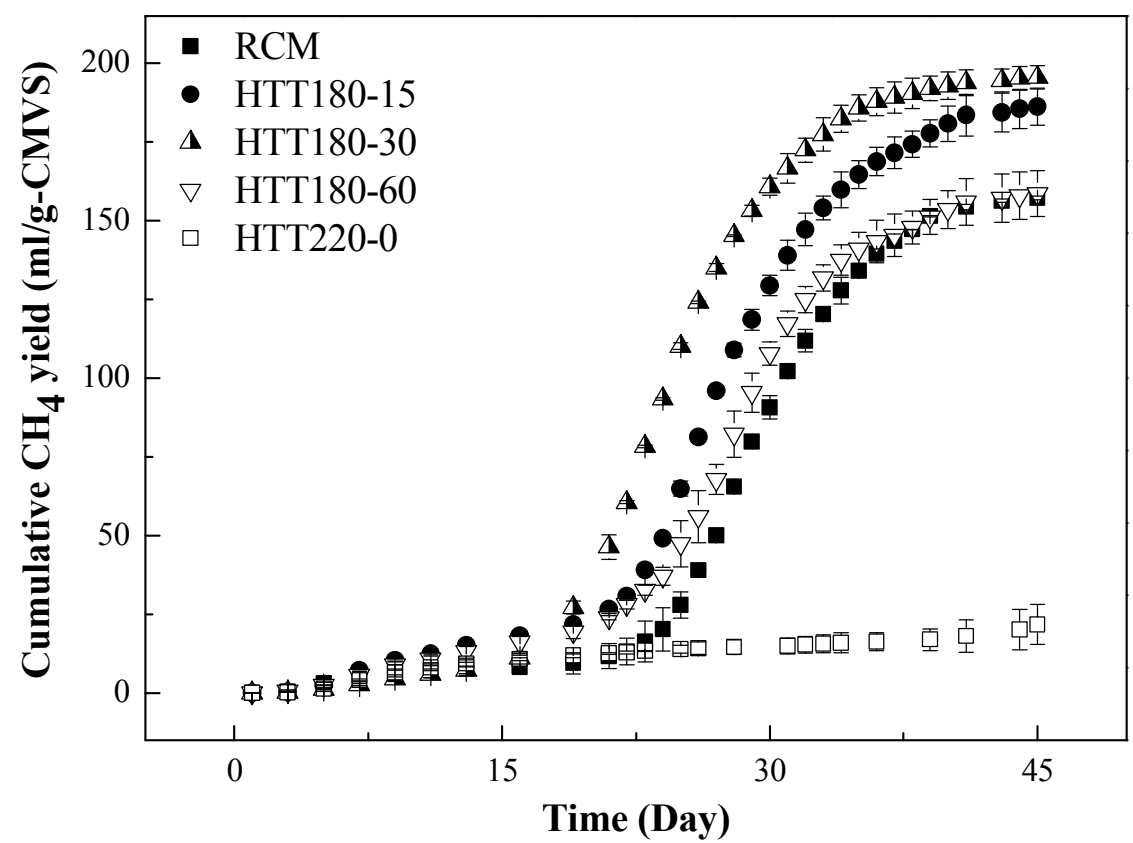

Figure S2. Cumulative $\mathrm{CH}_{4}$ production from non-treated RCM or the HTT processed and ammonia-stripped CM samples. 